\title{
Early polymerase chain reaction detection of Chagas disease reactivation in heart transplant patients
}

\author{
Priscilla Almeida da Costa, MS, ${ }^{a, 1}$ Marcela Segatto, PhD, ${ }^{\mathrm{b}, 1}$ \\ Danielle Fernandes Durso, PhD, ${ }^{a}$ Wagson José de Carvalho Moreira, BSc, ${ }^{a}$ \\ Lucas Lodi Junqueira, MD, PhD, ${ }^{\mathrm{C}}$ Fábio Morato de Castilho, MD, MS, ${ }^{\mathrm{C}}$ \\ Silvio Amadeu de Andrade, $M D{ }^{c}$ Cláudio Léo Gelape, MD, PhD, ${ }^{d}$ \\ Egler Chiari, $\mathrm{PhD},{ }^{\mathrm{e}}$ Andréa Teixeira-Carvalho, $\mathrm{PhD}{ }^{\dagger}$ \\ Sergio Danilo Junho Pena, MD, PhD, ${ }^{a}$ Carlos Renato Machado, PhD, ${ }^{a}$ \\ Gloria Regina Franco, PhD, ${ }^{a}$ Geraldo Brasileiro Filho, MD, PhD, ${ }^{g}$ \\ Maria da Consolação Vieira Moreira, MD, PhD, ${ }^{h}$ and Andréa Mara Macedo, PhD $^{a}$
}

From the ${ }^{a}$ Departamento de Bioquímica e Imunologia, Instituto de Ciências Biológicas, Universidade Federal de Minas Gerais, Belo Horizonte, Minas Gerais, Brazil; ${ }^{b}$ Departamento de Genética, Instituto de Biociências, Universidade Estadual Paulista, Botucatu, São Paulo, Brazil; ${ }^{c}$ Hospital das Clinicas, Universidade Federal de Minas Gerais, Belo Horizonte, Minas Gerais, Brazil; ${ }^{d}$ Departamento de Cirurgia, Faculdade de Medicina, Universidade Federal de Minas Gerais, Belo Horizonte, Minas Gerais, Brazil; ${ }^{e}$ Departamento de Parasitologia, Instituto de Ciências Biológicas,

Universidade Federal de Minas Gerais, Belo Horizonte, Minas Gerais, Brazil; ${ }^{f}$ Centro de Pesquisas René Rachou,

Fiocruz-Minas, Grupo Integrado de Pesquisas em Biomarcadores, Belo Horizonte, Minas Gerais, Brazil; ${ }^{g}$ Departamento de Patologia da Faculdade de Medicina, Universidade Federal de Minas Gerais, Belo Horizonte, Minas Gerais, Brazil; and the ${ }^{h}$ Departamento de Clinica Médica, Faculdade de Medicina, Universidade Federal de Minas Gerais,

Belo Horizonte, Minas Gerais, Brazil.

\section{KEYWORDS:}

Chagas disease reactivation; endomyocardial biopsy; heart transplantation; molecular diagnostics; polymerase chain reaction

\begin{abstract}
BACKGROUND: Heart transplantation is a valuable therapeutic option for Chagas disease patients with severe cardiomyopathy. During patient follow-up, the differential diagnosis between cardiac transplant rejection and Chagas disease infection reactivation remains a challenging task, which hinders rapid implementation of the appropriate treatment. Herein we investigate whether polymerase chain reaction (PCR) strategies could facilitate early detection of Trypanosoma cruzi ( $T$ cruzi) in transplanted endomyocardial biopsies (EMBs).

METHODS: In this study we analyzed 500 EMB specimens obtained from 58 chagasic cardiac transplant patients, using PCR approaches targeted to nuclear (rDNA 24S $\alpha$ ) and kinetoplastid (kDNA) markers, and compared the efficiency of these approaches with that of other tests routinely used.

RESULTS: T cruzi DNA was detected in 112 EMB specimens derived from 39 patients (67.2\%). The first positive result occurred at a median 1.0 month post-transplant. Conventional histopathologic, blood smear and hemoculture analyses showed lower sensitivity and higher median time to the first positive result. Patient follow-up revealed that 31 of 39 PCR-positive cases presented clinical reactivation of
\end{abstract}

\footnotetext{
${ }^{1}$ The first 2 authors (P.A.d.C. and M.S.) contributed equally to this work.

Reprint requests: Priscilla Almeida da Costa, MS, Departamento de Bioquímica e Imunologia, Instituto de Ciências Biológicas, Universidade Federal de Minas Gerais, Avenida Antonio Carlos 6627, Pampulha, Belo Horizonte, MG 31270901, Brazil. Telephone: +55-31-3409-2641. Fax: +55-31-3409-2984.

E-mail address: andrea@ufmg.br
} 
Chagas disease at different time-points after transplantation. PCR techniques showed considerable sensitivity (0.82) and specificity (0.60), with area under the receiver operating characteristic (ROC) curves of 0.708 ( $p=0.001)$. Moreover, PCR techniques anticipated the clinical signs of Chagas disease reactivation by up to 36 months, with a median time of 6 months and an average of 9.1 months.

CONCLUSIONS: We found a good association between the PCR diagnosis and the clinical signs of the disease, indicating that the PCR approaches used herein are suitable for early diagnosis of Chagas disease reactivation, with high potential to assist physicians in treatment decisions. For this purpose, an algorithm is proposed for surveillance based on the molecular tests.

J Heart Lung Transplant 2017;36:797-805

(C) 2017 International Society for Heart and Lung Transplantation. All rights reserved.

Chagas disease, caused by the protozoan Trypanosoma cruzi (T cruzi), is the third most common parasitic infection in the world after malaria and schistosomiasis, with 28,000 new cases and approximately 12,000 deaths each year in Central and South America. ${ }^{1,2}$ Increasing cases have been reported in the United States and Europe, mainly due to immigration, ${ }^{3-5}$ generating implications not only from an epidemiologic point of view but also for transplant centers.

In the acute phase of Chagas disease, most people present high parasitemia, fever, and other non-specific symptoms. About two thirds of those infected will evolve to a chronic asymptomatic phase, which lasts throughout their lifetime. The remaining one third will generally develop heart or gastrointestinal complications 10 to 30 years after the initial infection. Of these, approximately $30 \%$ develop the chronic form of Chagas heart disease, which can lead to heart failure or sudden death. ${ }^{6}$

Heart transplantation is the ultimate therapeutic option for patients with severe Chagas cardiomyopathy. ${ }^{7}$ However, the immunosuppressive treatment that accompanies transplant increases the likelihood of infections, including reactivation of Chagas disease..$^{8-10}$

The main drugs used for specific anti- $T$ cruzi treatment are benznidazole and nifurtimox; however, due to the occurrence of substantial side effects, the benefits of prophylactic use of these agents in transplanted patients just before or after transplantation remain to be determined. ${ }^{11}$

Microscopic examination of endomyocardial biopsy (EMB) is a widely accepted method for assessment of post-transplant myocardial injury, but differentiating between inflammation caused by immunologic rejection and Chagas disease reactivation is still a challenge. EMB histopathologic analysis, blood smear examination, xenodiagnosis and hemoculture are the methods currently used for the direct diagnosis of $T$ cruzi reactivation. Although highly specific, these methods have shown low sensitivity due to low circulating parasitemia and rare parasite nests (intracellular amastigotes) in heart tissues. In addition, both xenodiagnosis (diagnosis via detection of infective forms $T$ cruzi in the feces of triatomine bugs fed directly or indirectly on the patient's blood) and hemoculture take a long time to provide results. ${ }^{12,13}$ Serologic tests, on the other hand, are useful only for seronegative patients receiving organs from seropositive donors. Thus, it is crucial to develop a diagnosis method that can specifically detect Chagas disease reactivation in heart transplant patients.
We aimed to demonstrate that molecular diagnosis by polymerase chain reaction (PCR): (i) can successfully identify the presence of $T$ cruzi in EMB specimens of patients with chagasic cardiomyopathy submitted to heart transplant; and (ii) assists in early identification of patients at risk of developing reactivation of the infection. Ultimately, such information would help physicians to decide whether and at what point transplanted patients should receive anti- $T$ cruzi drug treatment.

\section{Methods}

\section{Patients and sample selection}

Our study protocol complied with the Helsinki Declaration and was approved by the institutional review board at the Universidade Federal of Minas Gerais (UFMG). All patients provided written informed consent.

A total of 58 consecutive adult chagasic patients were analyzed, all of whom underwent heart transplantation in the cardiology department of Hospital das Clínicas of the UFMG (HC-UFMG) during the period from 2008 to 2014. Each of these patients had at least 2 positive serology tests for Chagas disease.

\section{Post-operative immunosuppression}

All patients received standard therapy with calcineurin inhibitors (cyclosporine or tacrolimus), mycophenolate mofetil and prednisone. After 6 months, prednisone was weaned whenever possible.

\section{Post-operative EMB}

EMB was performed routinely according to Costanzo et al. ${ }^{14}$ For histopathologic study, 500 EMB specimens from all 58 patients were examined. A minimum of 4 endomyocardial fragments, 1 to $3 \mathrm{~mm}$ in diameter, were harvested under sterile conditions from the right ventricle. Two or 3 biopsy specimens were fixed in $4 \%$ buffered formaldehyde and embedded in paraffin. The remaining 2 specimens were quick frozen.

For molecular analyses, EMB specimens were grouped into 2 categories: (1) 155 samples obtained from the sample bank of the HC-UFMG that had been collected before beginning the present investigation, all of which were fixed in buffered formalin and then embedded in paraffin and then used for the retrospective analysis; and (2) 345 samples specifically collected for this work and preserved in absolute ethanol, then used in the prospective analysis. 


\section{Post-transplant follow-up}

After transplantation, all patients were followed regularly by a cardiologist and the monitoring of $T$ cruzi reactivation was performed routinely during monthly medical visits or whenever necessary during the first year, and then every 3 months and during suspected clinical or laboratory-supported reactivation episodes when there was:

1. Clinical suspicion of $T$ cruzi reactivation, defined as signs and symptoms similar to allograft dysfunction associated with episodes of fever, new skin lesions, arrhythmias or new conduction blocks on electrocardiography, left ventricular dysfunction on echocardiography or neurologic manifestations.

2. Positive results during laboratory monitoring, as detected by direct microscopy of blood smear, hemoculture or histopathologic or PCR analysis of the EMB.

Patients suspected of clinical reactivation underwent the following: skin biopsy in the presence of skin lesions; EMB if myocarditis was suspected; computed tomography scan; magnetic resonance imaging of the brain and cerebrospinal fluid analysis in the presence of neurologic manifestations; and myelogram or bone marrow biopsy in cases of suspected bone marrow involvement.

Detection of parasitemia in serial samples or presence of amastigotes in the implanted heart or other tissues confirmed reactivation.

Six patients died during the study: one due to acute graft failure, two due varicella pulmonary or cytomegalovirus infections, and two of unknown causes. Only one death was related to reactivation or complications associated with Chagas disease, and the cause of death was suspected to be neurologic reactivation of Chagas. No other loss occurred.

\section{Diagnostic techniques}

\section{Histopathology}

The degree of cellular rejection each the specimen was graded according to International Society for Heart Transplantation criteria. $^{15,16}$ At least 15 sections, stained with hematoxylin and eosin, from each endomyocardial or skin biopsy were carefully examined for inflammatory reaction and parasite nests.

\section{Hemoculture}

Peripheral blood $(30 \mathrm{ml})$ was collected into sodium heparin tubes and centrifuged at $4{ }^{\circ} \mathrm{C}$ to harvest the plasma. The packed cells were washed twice by centrifugation at $4^{\circ} \mathrm{C}$ in liver infusion tryptose (LIT) medium, distributed among 6 tubes containing $3 \mathrm{ml}$ of LIT, and incubated at $28^{\circ} \mathrm{C}$ in a biochemical oxygen demand incubator. Procedures were done in sterile conditions and processed $<6$ hours after blood was drawn. All tubes were mixed gently once per week and examined by microscopy, searching for motile trypanosomes once per for up to 3 months.

\section{Blood smear}

Blood smears were prepared from fresh ethylene-diamine tetraacetic acid (EDTA)-anti-coagulated blood. At least 400 microscopic fields $(1,000 \times$ magnification) were examined before the sample was declared free of the parasite.
Molecular diagnosis of $T$ cruzi

\section{Preparation of T cruzi genomic DNA}

DNA extraction was performed using tissue kits (QIAamp DNA FFPE or QIAamp DNA Mini Kit; Qiagen, Valencia, CA) for paraffin-embedded and fresh EMB specimens, respectively, according to the manufacturer's instructions.

As a control procedure and to verify DNA integrity after extraction, a polymorphic region of the human genome was amplified by PCR using the primers MID-768-F (5'-CATTACCAGTAGAGTGGGGA-3') and MID-768-R (5'-CTATGCCCTACTGGATCTAGG-3'), as described by Weber et al. ${ }^{17}$ A pool of human DNA was used as positive control and only the reagents of the PCR constituted the negative control.

\section{Mitochondrial DNA PCR}

$T$ cruzi DNA detection was accomplished by specific amplification of a 330-bp fragment corresponding to the variable region of the mitochondrial (kDNA) minicircle. Each reaction with a final volume of $20 \mu \mathrm{l}$ was composed of $1.5 \mathrm{mmol} / \mathrm{liter} \mathrm{MgCl}_{2}$, Green Go Taq buffer (Promega, Madison, WI), $250 \mathrm{mmol} / \mathrm{liter}$ of each deoxynucleotide triphosphate, $1 \mathrm{mmol} / \mathrm{liter}$ of primers S35 (5'-AAATAATGTACGGGKGAGATGCATGA-3') and S36 (5'-GGTTCGATTGGGGTTGGTGTAATATA-3'), ${ }^{18} 1$ U Go Taq Flexi DNA polymerase (Promega) and $3 \mu$ of total extracted DNA.

Reaction conditions were initial denaturation at $94^{\circ} \mathrm{C}$ for 5 minutes, followed by 35 cycles of annealing at $60^{\circ} \mathrm{C}$, extension at $72^{\circ} \mathrm{C}$, denaturation at $94^{\circ} \mathrm{C}$ for 1 minute for each step and a final extension to 10 minutes. Five microliters of each PCR product was subjected to electrophoresis in $6 \%$ polyacrylamide gel and then silver stained. ${ }^{19}$ DNA of $T$ cruzi JG strain was included as a positive control and the negative control comprised all PCR reagents without DNA addition.

\section{Real-time PCR of 24So rDNA}

The protocol was performed as described by Freitas and colleagues based on a hemi-nested PCR amplification of the D7 region of $24 \mathrm{~S} \alpha$ ribosomal DNA (rDNA), ${ }^{20}$ followed by identification of the products using real-time PCR denaturation curves. In the first round of amplification, $5 \mu \mathrm{l}$ of the total extracted DNA was used as template and D75 (5'-CAGATCTTGGTTGGCGTAG-3') and D72 $\left(5^{\prime} \text {-TTTTCAGAATGGCCGAACAGT- } 3^{\prime}\right)^{21}$ as primers. A second round was performed in real time (ABI7900; Applied Biosystems) using SYBR Green PCR Master Mix (Applied Biosystems), primers D71 (5'-AAGGTGCGTCGACAGTGTGG-3' $)^{21}$ and D72 and $2 \mu \mathrm{l}$ of the first PCR product as template. DNA of $T$ cruzi JG strain and clone Col1.7G2 were included as positive control, whereas components of the PCR reaction with no DNA were used as negative control.

Primers used in both molecular methodologies were designed to match conserved regions of parasite DNA, being able to amplify the target DNA in all T cruzi populations.

\section{Statistical analysis}

The kappa index was calculated to evaluate the correlation between the 2 PCRs used for molecular diagnosis. Values $>0.75$ were considered indicative of excellent agreement, 0.75 to 0.40 as good 
to reasonable and $<0.40$ as poor. ${ }^{22} p<0.05$ was considered statistically significant.

The MedCALC program was used to build receiver operating characteristic (ROC) curves, according to DeLong et al. ${ }^{23}$ For analysis of specificity, sensitivity and area under the ROC curve, positive results (from PCR or histopathology analyses) were denoted by 1 and negative results by 0 , with $>0$ selected as the criterion value. We used the clinical manifestation of patients as the "gold standard" reference. Significant $p$-values $(p<0.05)$ indicated that that area under the ROC curve could statistically distinguish positive and negative groups.

\section{Results}

Using 2 molecular approaches and conventional histopathology, we analyzed 500 samples of EMB specimens collected from 58 chagasic patients submitted to heart transplantation at the HC-UFMG. Some of these patients also had samples analyzed by hemoculture and blood smear. Clinical follow-up was completed in all of the patients.

\section{Molecular analysis}

Using a conventional PCR targeted to $T$ cruzi $\mathrm{kDNA}$, we identified the positive samples by the presence of 330- and 660-bp bands, which correspond to 1 or 2 contiguous variable regions of the minicircles (Figure 1A). We also performed quantitative PCR to amplify the $T$ cruzi rDNA $24 \mathrm{~S} \alpha$, and used the melting-curve program to distinguish the infected tissues qualitatively. The positive results were those presenting high amplification peaks with a melting temperature (MT) between $76^{\circ}$ and $81^{\circ} \mathrm{C}$, whereas the negative results and unspecific products showed irregular peaks exhibiting MT outside of this range (Figure 1B).

Thirty-nine of the 58 patients assessed (67.2\%) were positive on at least 1 of the 2 molecular tests performed at different time-points post-transplant. Considering the results from both methods, on average, these positive patients took $3.1 \pm 4.4$ months post-transplant (median 1 month) to show their first positive EMB, with a range of 7 days to 20 months (Table 1).

We compared the sensitivity of the 2 molecular approaches in detecting $T$ cruzi DNA in the EMB specimens. Although kDNA PCR proved to have greater sensitivity compared with rDNA 24S $\alpha$ quantitative PCR (102 positive samples from 36 patients vs 73 samples from 30 patients, respectively), there was good correlation between the results generated by both approaches, as demonstrated by the correlation index kappa $=0.663$ and $p<0.001$. Moreover, a combination of the techniques was essential to diagnose all of the positive samples. Using only kDNA PCR, 3 (10\%) of the 30 infected patients would be misidentified as

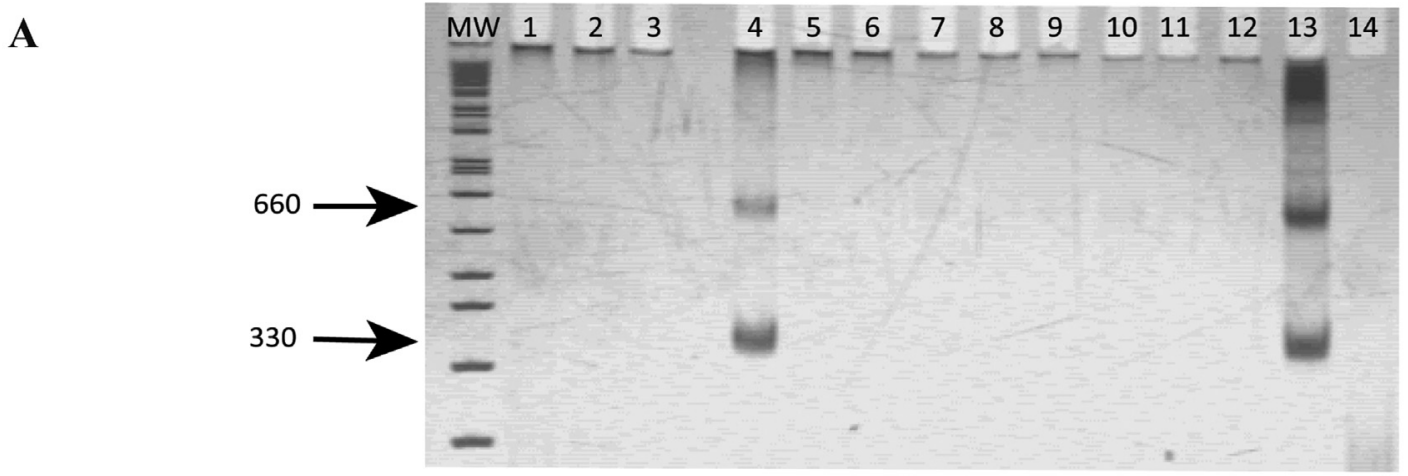

B

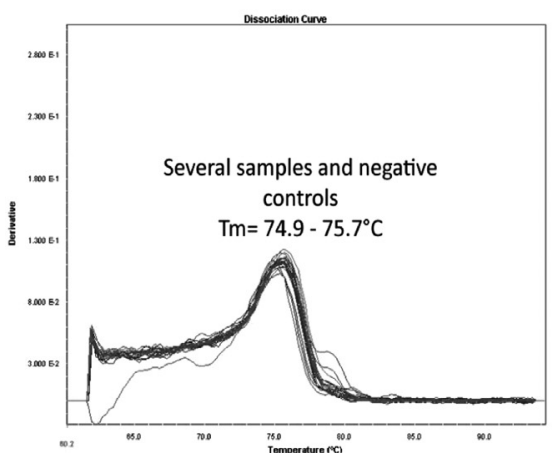

II

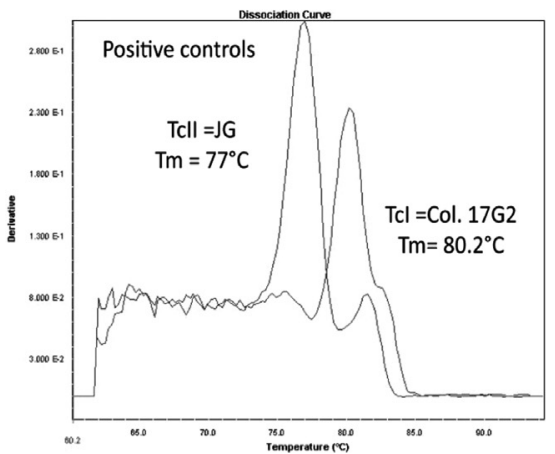

III

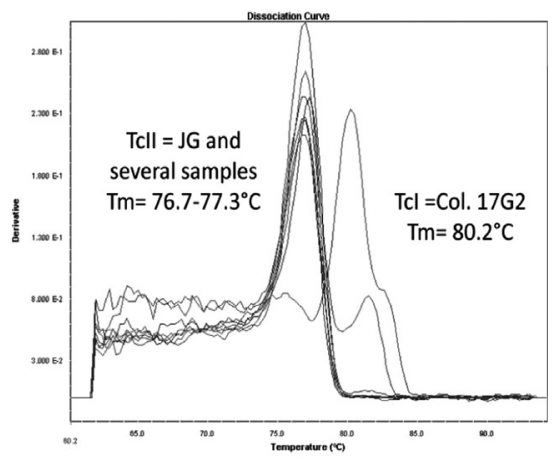

Figure 1 Representative results of the PCR techniques. (A) Amplification of variable 330/660-bp regions of the T cruzi kDNA minicircle from endomyocardial biopsies of heart transplant patients: 1 to 12: EMB specimens of patients, where 4 showed positive amplification products; 13: DNA from T cruzi JG strain (positive control); 14: no DNA template (negative control). MW, standard molecular weight (1-kb Plus DNA Ladder; Invitrogen). (B) Dissociation curves obtained by the melting curve program for the rDNA $24 \mathrm{~S} \alpha$ gene from biopsies of heart transplant patients. (I) Unspecific peaks obtained from negative EMB specimens in contrast to negative controls (without DNA). (II) Typical peaks derived from $T$ cruzi DNA of JG (TcII) and Col1.7G2 (TcI) populations (positive controls). (III) Peaks obtained from positive EMB specimens in contrast to positive controls. 
Table 1 Percentage of Positive Results and Time After Transplantation of the First Positive Result (Average and Median Times) for Each Test.

\begin{tabular}{lllll}
\hline Test & Number of patients & Positive patients & Average time (months) & Median time (months) \\
\hline Molecular diagnostic (EMB) & 58 & $65.5 \%$ & $3.1 \pm 4.4$ & 1.0 \\
Histopathology (EMB) & 58 & $17.2 \%$ & $11.6 \pm 11.07$ & 5.5 \\
Blood smear & 42 & $26.2 \%$ & $3.5 \pm 2.87$ & 3.0 \\
Hemoculture & 29 & $20.7 \%$ & $6.8 \pm 5.11$ & 6.5 \\
\hline
\end{tabular}

EMB, endomyocardial biopsy.

negatives, whereas the exclusive use of rDNA $24 \mathrm{~S} \alpha$ quantitative PCR would fail to detect the $T$ cruzi in $25 \%$ of the kDNA-positive patients (Table 2).

\section{Other parasitologic analyses}

All 500 EMB specimens were also submitted to histopathologic analysis, where $T$ cruzi was found in 10 patients $(17.2 \%)$ at $11.6 \pm 11.07$ months post-transplant (median 5.5 months) (Table 1).

Some patients were further submitted to direct analysis of blood smear exam (89 samples from 42 patients) and hemoculture (50 samples from 29 patients). Although these analyses were performed after patients presented the first clinical signs of Chagas disease reactivation, only $26.2 \%$ and $20.7 \%$ of patients analyzed were positive for blood smear or hemoculture, respectively, on direct examination. On average, these techniques were able to detect $T$ cruzi after 3.5 or 6.8 months post-transplant, respectively (Table 1).

\section{Molecular analyses and clinical follow-up comparison}

During follow-up of all 58 patients, 38 presented with clinical reactivation of Chagas disease at different time-points post-surgery. The main clinical signs were skin lesions (panniculitis with $T$ cruzi amastigotes) and

Table 2 Comparison of Contingency of Results for 2 PCR Methods of Molecular Diagnostics of $T$ cruzi Reactivation After Heart Transplant.

\begin{tabular}{|c|c|c|c|}
\hline & \multirow[b]{2}{*}{ kDNA PCR } & \multicolumn{2}{|c|}{ rDNA quantitative PCR } \\
\hline & & Positive & Negative \\
\hline \multirow[t]{2}{*}{ Samples $^{a}$} & Positive & 63 & 39 \\
\hline & Negative & 10 & 388 \\
\hline \multirow[t]{2}{*}{ Patients $^{b}$} & Positive & 27 & 9 \\
\hline & Negative & 3 & 19 \\
\hline
\end{tabular}

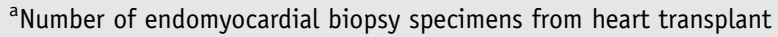
patients with chagasic cardiopathy presenting positive or negative results for the polymerase chain reaction (PCR) methods tested (kappa $_{\text {samples }}=0.668, p<0.001$, chi-square test).

${ }^{\mathrm{b}}$ Number of heart transplant patients with chagasic cardiopathy presenting positive or negative results for the PCR methods tested (kappa $_{\text {patients }}=0.653 ; p<0.001$, chi-square test). myocarditis. Two patients presented with signs of neurologic reactivation of Chagas disease.

Patients performed, on average, 5 post-transplant echocardiograms during the study period. The mean of left ventricular ejection fraction (LVEF) in these exams was $64 \%$ ( $21 \%$ to $87 \%$ ). LVEF $>50 \%$ may be considered normal or preserved and $<50 \%$ may be considered reduced. Only 9 of the 58 patients presented at least 1 echocardiogram with LVEF $<50 \%$ and no link between reduction of LVEF and chagasic reactivation was detected.

Despite the aforementioned findings, there was a good association between the PCR results and clinical reactivation of the disease, as 31 of the 38 patients with clinical reactivation (81.6\%) also showed positive results for at least 1 of the molecular tests (Figure 2).

Taking together the results of both PCR techniques, the molecular assay used presented considerable sensitivity $(0.82)$ and specificity (0.60). In contrast, only 10 of the 38 (26\%) patients with Chagas disease reactivation were diagnosed by histopathologic analysis of their EMB, showing lower sensitivity (0.26). Areas under the ROC curve for molecular and histopathologic assessment of EMBs from all 58 patients were 0.708 and 0.632 , respectively (Figure $3 \mathrm{~A}$ ).

When we compared the results obtained only from those patients who underwent all 4 diagnostic methods ( 29 of 58 patients), the molecular diagnosis showed a sensitivity of 0.79 , whereas histopathology, blood smear and hemoculture

Clinical reactivation of Chagas disease

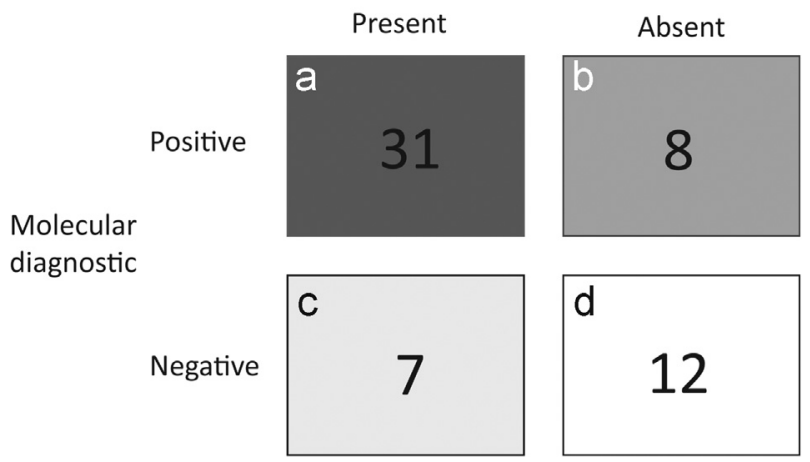

Figure 2 Comparison of reactivation results between clinical and PCR molecular diagnostic procedures using EMB specimens (paraffin-embedded or fresh biopsies) from heart transplant patients with chagasic cardiopathy. (1) ${ }^{\mathrm{a}}$ True positive; ${ }^{\mathrm{b}}$ false positive; ${ }^{\mathrm{c}}$ false negative; ${ }^{d}$ true negative; (2) $N=58$; (3) total number of positive tests $(a+b)=39$; (4) total number of clinical reactivations $(a+c)=38$. 
ROC curves for comparisons
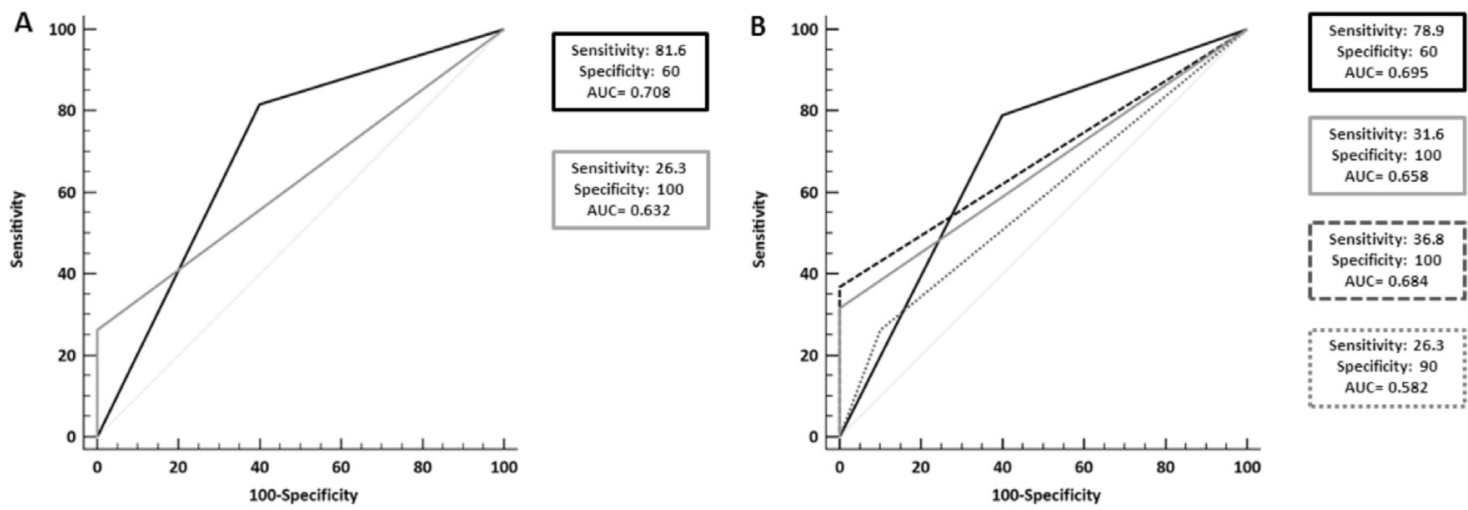
Specificity: 100 AUC $=0.684$

- Molecular diagnostic (EMB)

- Histopathology (EMB)

---- Blood smear ….... Hemoculture

Figure 3 ROC curves for different diagnosis approaches compared with clinical reactivation of Chagas disease. (A) Molecular diagnosis and histopathologic analysis of 500 EMB specimens from 58 patients (B) Molecular diagnosis, histopathologic analysis, blood smear and hemoculture of samples from 29 patients. Criterion $>0$ (positive $=1$, negative $=0$ ); AUC, area under the receiver operating characteristic (ROC) curve $(p \leq 0.001)$.

showed sensitivities of $0.32,0.37$ and 0.26 , respectively. The areas under the ROC curves for this subgroup of patients for molecular diagnosis, histopathology, blood smear and hemoculture were $0.695,0.658,0.684$ and 0.582 , respectively, indicating that the molecular method described herein is more efficient at detecting $T$ cruzi in patient samples (Figure 3B).

To assess whether both PCR methodologies could equally anticipate the clinical diagnosis of Chagas disease reactivation, we performed a retrospective study in which we analyzed EMB specimens embedded in paraffin blocks from patients transplanted at least 5 years early. For this analysis, the first 6 biopsies of 24 patients were compared with clinical data regarding disease reactivation (Table 3). In general, the sooner the biopsy samples showed PCRpositive results, the earlier the clinical reactivation of the disease occurred. Patients who had clinical reactivation before 1 year post-transplant had the first positive biopsy $<1$ month after surgery. The combined PCR techniques could anticipate the clinical signs of Chagas disease reactivation by 1.5 to 36 months, with a median time of 6 months (9.1 months, on average). When using only 1 of the molecular techniques, the average time increased to 9.8 or 10.9 months, with a median of 6 months and 8.5 months for kDNA PCR and $24 \mathrm{~S} \alpha$ quantitative PCR, respectively. These data suggest that both molecular diagnostics proposed can successfully anticipate Chagas disease reactivation.

Taken together, our results allowed us to propose the algorithm illustrated in Figure 4 for managing chagasic patients submitted to EMB after heart transplantation. This algorithm aims to help physicians decide when and which treatment to adopt, considering that differential diagnosis of clinical rejection and/or Chagas disease reactivation remains a challenge. The algorithm also represents a simple and logical way to follow-up patients with positive or negative results for histopathologic analysis of EMB, molecular diagnosis and/or clinical signs of Chagas disease reactivation.
Different combinations of positive results for these parameters can indicate cases for specific anti- $T$ cruzi treatment (we found 46 patients in this situation). Inflammation of the cardiac tissue associated with consistently negative results of reactivation of $T$ cruzi infection suggests transplant rejection (12 of our patients belong to this group). On the other hand, a lack of inflammation of cardiac tissue, absence of clinical signs and negative histopathologic and PCR findings would suggest a cure of chagasic myopathy, or at least wellcontrolled cardiac Chagas disease; however, no patient in our study fit this description (Figure 4).

\section{Discussion}

Heart transplantation is a valuable therapeutic tool for patients with severe cardiac Chagas disease. However, determining whether inflammatory signs present in EMB specimens of these patients are due to heart rejection and/or $T$ cruzi infection reactivation is a difficult task. This is attributable to several factors: (i) inflammatory infiltrates found in EMB as result of Chagas disease reactivation are similar to those triggered by cardiac transplant rejection ${ }^{24}$; (ii) parasite nests are rarely found by histopathologic examination $^{25}$; (iii) conventional parasitologic methods have low sensitivity due to low parasitemia observed in the chronic phase of disease ${ }^{26}$; and (iv) serologic tests are not useful except for monitoring seronegative patients receiving organs from seropositive donors. Therefore, developing a molecular method able to detect Chagas disease reactivation in transplanted patients would clearly help to overcome all of the aforementioned shortcomings.

PCR-based methods have increasingly been used for Chagas disease diagnosis, being considered more sensitive than pathologic analyses. The main targets of PCR assays are satellite or minicircle DNA, ${ }^{27-33}$ both of which are present in multiple copies in the genome of the parasite genome. ${ }^{33,34}$

Several studies have shown that amplification of the variable region of $T$ cruzi minicircle $\mathrm{kDNA}$, the same 
Table 3 PCR Analysis of $T$ cruzi Obtained From the First 6 Endomyocardial Biopsies Performed After Heart Transplantation of 24 Patients With Chagasic Cardiopathy.

\begin{tabular}{|c|c|c|c|c|c|c|c|}
\hline \multirow[b]{2}{*}{ Patient no. ${ }^{a}$} & \multirow{2}{*}{$\begin{array}{l}\text { Date of clinical reactivation of } \\
\text { Chagas disease }^{b}\end{array}$} & \multicolumn{6}{|c|}{ kDNA and rDNA PCR results/days after transplant ${ }^{\mathrm{C}}$} \\
\hline & & 7 & 14 & 21 & 28 & 45 & 60 \\
\hline 1 & Several episodes of reactivation & $+^{d}$ & $+^{\mathrm{d}}$ & $-{ }^{\mathrm{e}}$ & - & - & - \\
\hline 2 & 2 months & - & $+^{f}$ & - & - & - & $+^{f}$ \\
\hline 3 & 2 months & - & $+^{g}$ & - & - & - & - \\
\hline 4 & 3 months & $+^{f}$ & $+^{f}$ & - & $+^{f}$ & $+^{f}$ & - \\
\hline 5 & 3 months & - & $+^{f}$ & - & $+^{g}$ & - & - \\
\hline 6 & 4 months & $+^{f}$ & $t^{d}$ & - & - & - & $+^{\mathrm{d}}$ \\
\hline 7 & 4 months & - & - & - & $t^{f}$ & - & $+^{f}$ \\
\hline 8 & 6 months & $+^{f}$ & $+^{f}$ & $+^{f}$ & - & - & $+^{f}$ \\
\hline 9 & 6 months & - & - & - & - & - & - \\
\hline 10 & 9 months & - & $+^{d}$ & $+^{d}$ & - & - & $+^{d}$ \\
\hline 11 & 10 months & - & - & $+^{g}$ & $+^{d}$ & $+^{d}$ & $+^{d}$ \\
\hline 12 & 17 months & - & $+^{d}$ & - & $t^{f}$ & $+^{d}$ & $+^{f}$ \\
\hline 13 & 18 months & - & - & - & - & - & - \\
\hline 14 & 20 months & - & - & - & - & - & $+^{f}$ \\
\hline 15 & 20 months & - & - & - & - & - & - \\
\hline 16 & 36 months & $+^{f}$ & - & - & $+^{d}$ & - & $+^{d}$ \\
\hline 17 & $\begin{array}{l}\text { No clinical reactivation (died from pulmonary } \\
\text { infection and varicella) }\end{array}$ & $+^{g}$ & $+^{f}$ & - & $+^{f}$ & - & $+^{d}$ \\
\hline 18 & No clinical reactivation (death from unknown causes) & - & - & $++^{f}$ & - & $+^{f}$ & $t^{f}$ \\
\hline 19 & No clinical reactivation & $+^{f}$ & - & $+{ }^{f}$ & $t^{f}$ & - & - \\
\hline 20 & No clinical reactivation & $+^{f}$ & $+^{f}$ & $+^{f}$ & $t^{f}$ & $+^{f}$ & $t^{f}$ \\
\hline 21 & No clinical reactivation & - & - & - & - & - & - \\
\hline 22 & No clinical reactivation & - & - & - & - & - & - \\
\hline 23 & No clinical reactivation & - & - & - & - & - & - \\
\hline 24 & No clinical reactivation & - & - & - & - & - & - \\
\hline \multicolumn{8}{|c|}{ 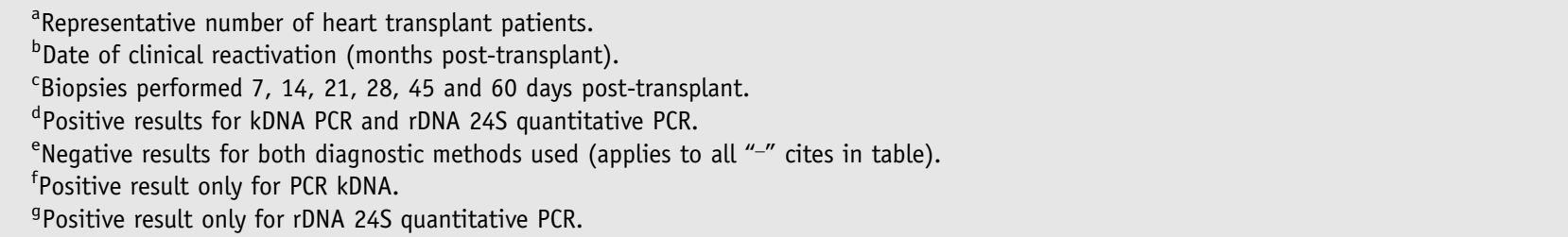 } \\
\hline
\end{tabular}

we used here, is very specific for detection of this parasite, , $29,34-37$ yet others have argued against this specificity. $^{38-40}$

The usefulness of PCR techniques to specifically evaluate for the presence of $T$ cruzi in EMB specimens has also been addressed in various studies. 9,29,39,40 However, all of these studies were conducted with small sample sizes (EMB specimens and patients) and short follow-up periods, which may have interfered with the findings. For instance, kDNA PCR was disallowed as a diagnosis strategy in a previous work, as some patients without clinical signs of Chagas disease reactivation were positive for this marker. However, because these patients were followed-up for only 12 months, it was not possible to conclude whether these discrepant data represented false positive PCR results or anticipation of clinical reactivation of the disease. ${ }^{40}$

To solve the debate about the usefulness of PCR to diagnose $T$ cruzi reactivation in EMB specimens of heart transplant patients, we used a combination of kDNA PCR and rDNA 24S $\alpha$ quantitative PCR in a larger sample for comparison with previous studies. Thus, our strategy not only increased the statistical power, but also the sensitivity, without losing the specificity of these methods, as we observed a good association between the molecular and clinical diagnoses of Chagas disease reactivation. For instance, a total of 352 EMB specimens obtained from 47 patients had inflammation without nesting of $T$ cruzi, as revealed by conventional histopathologic analysis, which could be understood as indicative of rejection. However, $T$ cruzi DNA was detected by PCR in 76 EMB specimens obtained from 29 of these patients, 21 of whom also presented clinical signs of Chagas disease reactivation at different time-points post-transplant. These data support the utility of the molecular tests employed herein to diagnose Chagas disease reactivation from biopsies of heart transplant patients with chagasic cardiopathy.

Analysis of the first 6 follow-up EMBs by kDNA PCR and rDNA $24 \mathrm{~S} \alpha$ quantitative PCR revealed that our strategy was able to anticipate clinical diagnosis of Chagas disease reactivation by up 36 months (median time of 6 months). Thus, we suggest that inclusion of these molecular tests in the clinical routine would help physicians to decide whether and when to introduce anti-Chagas therapy, as revealed by the algorithm proposed. This decision is important due 


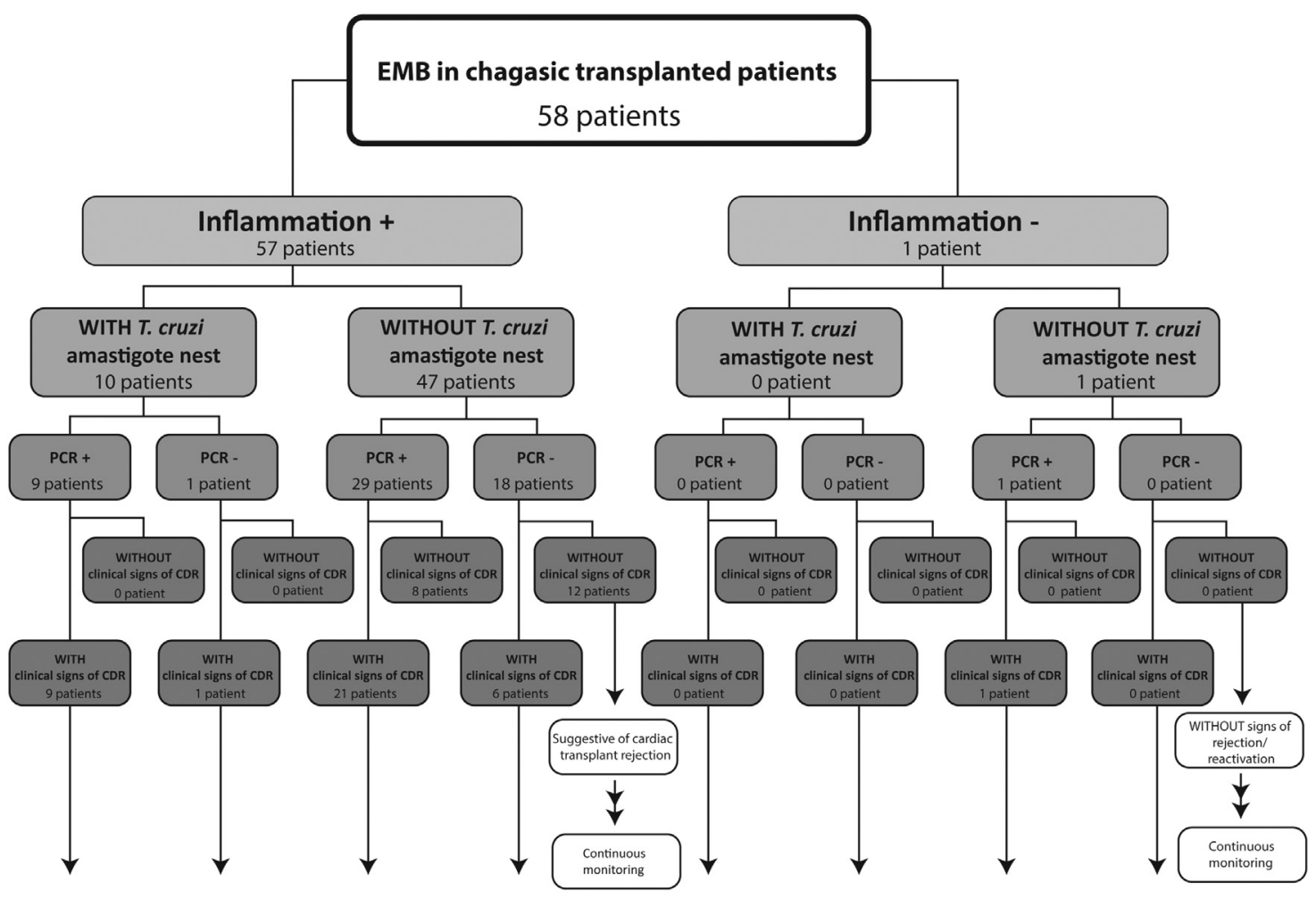

Indicative of specific treatment for T. cruzi

Figure 4 Proposed algorithm for managing chagasic patients submitted to EMB. Number of patients associated to each box represents the number of cases observed in the present study. CDR, Chagas disease reactivation; EMB, endomyocardial biopsy.

to the delicate health and immunologic status of most heart transplant patients after surgery.

Since 2008, we have followed 58 patients enrolled in this study by analyzing their EMB samples through the PCRs described. Two groups of positive patients were identified: (i) those with positive molecular diagnosis accompanied by clinical signs of Chagas disease reactivation $(n=31)$; and (ii) those with a positive molecular diagnosis but without signs of clinical reactivation $(n=8)$. The first group supports the suitability of this molecular strategy in the setting of Chagas disease reactivation. At first sight, the second group could represent false positives. However, because the PCRs employed here seem able to anticipate the clinical diagnosis, it is possible that these "false positives" are in fact patients who will present clinical manifestations of Chagas disease reactivation in the future. For these patients, continuous monitoring can confirm the benefit of early introduction of anti-Chagas drugs.

In conclusion, in this study we have evaluated the use of PCR for early diagnosis of Chagas disease reactivation in EMB specimens from heart transplant patients with chagasic cardiopathy. We found that the PCR techniques used can be employed as a valuable tool for differential diagnosis between parasite reactivation and cardiac transplant rejection. Also, this strategy succeeds in anticipating the diagnosis of clinical reactivation by up to several months. The relevant aspects of the proposed molecular diagnosis are its good sensitivity and specificity and its time efficiency. Indeed, the time required for reaching a PCR diagnosis is less than that required for standard parasitologic methods.

Future studies comparing the outcomes of chagasic heart transplant patients treated with anti-Chagas drugs based exclusively on the positive PCR result versus those treated only when patent parasitemia or clinical manifestations of reactivation are detected may confirm the clinical value of the proposed management strategy. We believe that implementation of this PCR diagnosis in the clinical setting will help physicians to determine the point at which heart transplant patients should begin receiving anti-chagasic drugs, a decision that will ultimately contribute to the wellbeing and survival of these patients.

\section{Disclosure statement}

The authors have no conflicts of interest to disclose. This work was supported by grants and fellowships from Fundação de Amparo a Pesquisa no Estado de Minas Gerais (FAPEMIG, Brazil), Conselho Nacional de Pesquisa e Desenvolvimento (CNPq, Brazil), Departamento de Ciência e Tecnologia do Ministério da Saúde (Decit/SCTIE/MS) and Coordenação de Aperfeiçoamento de Pessoal de Nível Superior (CAPES, Brazil).

\section{References}

1. World Health Organization. Chagas disease (American trypanosomiasis). Wkly Epidemiol Rec 2010;85:334-6.

2. World Health Organization. Making health research work for poor people, the world health report. TDR 2005:30-3. 
3. Milei J, Guerri-Guttenberg RA, Grana DR, et al. Prognostic impact of Chagas disease in the United States. Am Heart J 2009;157:22-9.

4. Guerri-Guttenberg RA, Ciannameo A, Di Girolamo C, et al. Chagas disease: an emerging public health problem in Italy? Infez Med 2009; 17:5-13.

5. Kransdorf EP, Czer LSC, Luthringer DJ, et al. Heart transplantation for Chagas cardiomyopathy in the United States. Am J Transplant 2013; 13:3262-8.

6. Manzullo EC, Chuit R. Risk of death due to chronic chagasic cardiopathy. Mem Inst Oswaldo Cruz 1999(suppl I):317-20.

7. Bocchi EA, Fiorelli A. The paradox of survival results after heart transplantation for cardiomyopathy caused by Trypanosoma cruzi. Ann Thorac Surg 2001;71:1833-8.

8. Godoy HL, Guerra CM, Viegas RF, et al. Infections in heart transplant recipients in Brazil: the challenge of Chagas' disease. J Heart Lung Transplant 2010;29:286-90.

9. Schijman AG, Vigliano C, Burgos J, et al. Early diagnosis of recurrence of Trypanosoma cruzi infection by polymerase chain reaction after heart transplantation of a chronic Chagas' heart disease patient. J Heart Lung Transplant 2000;19:1114-7.

10. Bacal F, Silva CP, Pires PV, et al. Transplantation for Chagas' disease: an overview of immunosuppression and reactivation in the last two decades. Clin Transplant 2010;24:29-34.

11. Viotti R, Vigliano C, Lococo B, et al. Side effects of benznidazole as treatment in chronic Chagas disease: fears and realities. Expert Rev Anti Infect Ther 2009;7:157-63.

12. Bacal F, Souza-Neto JD, Fiorelli AI, et al. II Diretriz Brasileira de Transplante Cardíaco. Arq Bras Cardiol 2009;94:e16-73.

13. Bulcão AA, Portela-Lindoso AA, Shikanai-Yasuda MA. Chronic Chagas' disease: from xenodiagnosis and hemoculture to polymerase chain reaction. Rev Saude Publica 2003:37:107-15.

14. Costanzo MR, Dipchand A, Starling R, et al. The International Society for Heart and Lung Transplantation guidelines for the care of heart transplant recipients. J Heart Lung Transplant 2010;29:914-56.

15. Billingham ME, Cary NRB, Hammond ME, et al. A working formulation for the standardization of nomenclature in the diagnosis of heart and lung rejection. J Heart Lung Transplant 1990;9:587-93.

16. Stewart S, Gayle L, Fishbein MC, et al. Revision of the 1990 working formulation for the standardization of nomenclature in the diagnosis of heart rejection. J Heart Lung Transplant 2006;25:1710-20.

17. Weber JL, David D, Heil J, et al. Human diallelic insertion/deletion polymorphisms. Am J Hum Genet 2002;71:854-62.

18. Wincker P, Britto C, Pereira JB, et al. Use of a simplified polymerase chain reaction procedure to detect $T$. cruzi in blood samples from chronic chagasic patients in a rural endemic area. Am J Trop Med Hyg 1994;51:771-7.

19. Santos FR, Epplen JT, Pena SD. Testing deficiency paternity cases with a Y-linked tetranucleotide repeat polymorphism. EXS 1993;67: 261-265.

20. Freitas JM, Lages-Silva E, Crema S, et al. Real time PCR strategy for the identification of major lineages of Trypanosoma cruzi directly in chronically infected human tissues. Int J Parasitol 2005;35:411-7.

21. Souto RP, Zingales B. Sensitive detection and strain classification of Trypanosoma cruzi by amplification of a ribosomal RNA sequence. Mol Biochem Parasitol, 62; 45-52.

22. Fleiss JL. Statistical methods for rates and proportions. 2nd ed. New York: John Wiley \& Sons; 1981.

23. DeLong ER, DeLong DM, Clarke-Pearson DL. Comparing the areas under two or more correlated receiver operating characteristic curves: a nonparametric approach. Biometrics 1988;44:837-45.
24. Andrade JA, Marin-Neto JA, Paola AAV, et al. I Diretriz latino americana para o diagnóstico e tratamento da cardiopatia chagásica. Arq Bras Cardiol 2011;97:1-48.

25. Benvenuti LA, Roggério A, Sambiase NV, et al. Polymerase chain reaction in endomyocardial biopsies for monitoring reactivation of Chagas' disease in heart transplantation. A case report and review of the literature. Cardiovasc Pathol 2005;14:265-8.

26. Meira WSF, Galvão LMC, Gontijo ED, et al. Trypanosoma cruzi recombinant complement regulatory protein: a novel antigen for use in an enzyme-linked immunosorbent assay for diagnosis of Chagas' disease. J Clin Microbiol 2002;40:3735-40.

27. Brasil PE, De Castro L, Hasslocher-Moreno AM, et al. ELISA versus PCR for diagnosis of chronic Chagas disease: systematic review and meta-analysis. BMC Infect Dis 2010;10:337.

28. Dias JCP. Epidemiology of Chagas disease. In: Wendel S, Brener Z, Camargo ME, editors. Chagas' disease-American trypanosomiasis: its impact on transfusion and clinical medicine. Sao Paulo: ISBT; 1992. p. 49-80.

29. Diez M, Favaloro L, Bertolotti A, et al. Usefulness of PCR strategies for early diagnosis of Chagas' disease reactivation and treatment follow-up in heart transplantation. Am J Transplant 2007;7:1633-40.

30. Duffy T, Bisio M, Altcheh J, et al. Accurate real-time PCR strategy for monitoring bloodstream parasitic loads in chagas disease patients. PLoS Negl Trop Dis 2009;3:e419.

31. Moser DR, Kirchhoff LV, Donelson JE. Detection of Trypanosoma cruzi by DNA amplification using the polymerase chain reaction. J Clin Microbiol 1989;27:1477-82.

32. Schijman AG, Bisio M, Orellana L, et al. International study to evaluate PCR methods for detection of Trypanosoma cruzi DNA in blood samples from Chagas disease patients. PLoS Negl Trop Dis 2011;5:e931.

33. Gonzalez A, Prediger E, Huecas ME, et al. Minichromosomal repetitive DNA in Trypanosoma cruzi: its use in a high-sensitivity parasite detection assay. Proc Natl Acad Sci USA 1984;81:3356-60.

34. Sturm NR, Degrave W, Morel C, et al. Sensitive detection and schizodeme classification of Trypanosoma cruzi cells by amplification of kinetoplast minicircle DNA sequences: use in diagnosis of Chagas' disease. Mol Biochem Parasitol 1989;33:205-14.

35. Avila H, Goncalves AM, Nehme NS, et al. Schizodeme analysis of Trypanosoma cruzi stocks from south and central America by analysis of PCR amplified minicircle variable region sequences. Mol Biochem Parasitol 1990;42:175-87.

36. Gomes ML, Galvão LM, Macedo AM, et al. Chagas' disease diagnosis: comparative analysis of parasitologic, molecular, and serologic methods. Am J Trop Med Hyg 1999;60:205-10.

37. Maldonado C, Albano S, Vettorazzi L, et al. Using polymerase chain reaction in early diagnosis of reactivated Trypanosoma cruzi infection after heart transplantation. J Heart Lung Transplant 2004;23: $1345-8$.

38. Castro AM, Luquetti AO, Rassi A, et al. Blood culture and polymerase chain reaction for the diagnosis of the chronic phase of human infection with Trypanosoma cruzi. Parasitol Res 2002;88:894-900.

39. Virreira M, Torrico F, Truyens C, et al. Comparison of polymerase chain reaction methods for reliable and easy detection of congenital Trypanosoma cruzi infection. Am J Trop Med Hyg 2003;68: $574-82$.

40. Benvenuti LA, Roggério A, Coelho G, et al. Usefulness of qualitative polymerase chain reaction for Trypanosoma cruzi DNA in endomyocardial biopsy specimens of chagasic heart transplant patients. J Heart Lung Transplant 2011;30:799-804. 